\title{
IS ‘ASSISTED REPRODUCTION’ REPRODUCTION?
}

\author{
Monika Piotrowska
}

\begin{abstract}
With an increasing number of ways to 'assist' reproduction, some bioethicists have started to wonder what it takes to become a genetic parent. It is widely agreed that sharing genes is not enough to substantiate the parent-offspring relation, but what is? Without a better understanding of the concept of reproduction (and what it means to say that an organism has reproduced) our thinking about parent-offspring relations and the ethical issues surrounding them risk being unprincipled. Here, I address that problem by offering a principled account of reproduction — the Overlap, Development and Persistence (ODP) account-which I believe best captures the meaning of 'genetic parenthood'.

\section{Keywords}

bioethics, genetic parenthood, mitochondrial donation, philosophy of biology, reproduction
\end{abstract}


Kinship was once understood as the cultural elaboration of biological facts, with the assumption that the biological facts were given and universally the same... From one perspective this is surely the case. But which 'biological facts' become socially relevant and the value or significance placed on them cannot be assumed. (Edwards 2014: 46)

Ethical discussion often draws on contentious conceptual interpretations of apparently biological facts. (Lewens 2015: 3)

\section{Introduction}

The primary motivation behind assisted reproduction is to have a child genetically related to oneself, to have a child of 'one's own'. This desire is apparently stronger than the financial costs and medical risks associated with assisted reproductive technologies. But some forms of assisted reproduction - known as 'third-party reproduction' - help individuals attain genetic parenthood by using genetic material from someone other than the parents intending to have the child ('intending parents' for short). For example, when an intending mother is known to have disease-linked mitochondrial DNA, a donor can contribute her healthy DNA to replace the unhealthy DNA of the intending mother. The result is a child with three genetic contributors: one male and two female. Rather curiously, however, the mitochondrial donor is required to acknowledge, by consent, that she will not be the child's genetic parent. ${ }^{1}$ In fact, the UK-based Nuffield Council on Bioethics conducted an inquiry into the ethical issues raised by mitochondrial donation and concluded that, "[this type of] donation does not indicate, either biologically or legally, any notion of the child having either a "third parent," or "second mother"' (Neuffield Council on Bioethics 2012: XVI). But this conclusion seems odd: if a child is 'one's own' in virtue of inheriting one's genetic material, why isn't the mitochondrial DNA donor one of the child's biological parents?

\footnotetext{
${ }^{1}$ See guidelines put forth by the Human Fertilisation and Embryology Authority (The Human Fertilisation and Embryology Authority 2015).
} 
Of course, we're used to thinking that children have only two parents (not three), but we're also used to thinking that reproduction involves the act of intercourse, fertilization, gestation and childbirth. Indeed, as that traditional way of thinking gets disrupted, one would expect our intuitions about parenthood to be disrupted as well, especially as our standard ideas about reproduction are being drastically altered. For example, in the arena of assisted reproduction, parts of the reproductive process can be bypassed, performed differently, or performed by different people. The end product might be the same, but as the process becomes less familiar, it's difficult to say who qualifies as a child's genetic parent. Without a better understanding of the concept of reproduction (and what it means to say that an organism has reproduced) our thinking about parent-offspring relations and the ethical issues surrounding them risk being unprincipled. Such manufactured answers may do for particular purposes, but they'll lack any coherent conceptual basis.

I'm not the first to notice this problem and to try to resolve it by thinking about the nature of reproduction. Heidi Mertes and Guido Pennings (2008) have offered an account of reproduction designed to discriminate between genetic parents and nonparents. Their account picks out familiar features of the reproductive process that, according to Mertes and Pennings, capture the correct meaning of 'genetic parenthood'. ${ }^{2}$ Mertes and Pennings' account also has the virtue of providing the Neuffield Council with a justification for rejecting the idea that a mitochondrial DNA donor qualifies as a genetic parent. Even so, I believe their account is mistaken. In this paper, I aim to show why and to offer an alternative, which will be better

\footnotetext{
${ }^{2}$ The conceptual relations between 'genetic parenthood', 'genetic offspring', and 'reproduction' are what is at issue. The fact that three concepts are in play gives the theorist options when considering how best to capture those relations. One could, for example, begin by trying to understand 'genetic parenthood' and draw from that understanding implications about 'reproduction' and 'genetic offspring.' This seems to be the approach of Mertes and Pennings. In contrast, my approach aims to settle problems about the parent-offspring relation by better understanding 'reproduction'. Obviously, theorists may take different approaches. Whatever approach is taken, however, the concepts of 'genetic parenthood', genetic offspring', and 'reproduction' should travel together, be congruent with the biological facts, and thereby capable of making sense of reproduction as a general concept.
} 
equipped to address both the Neuffield Council's concerns as well as broader concerns about the meaning of reproduction.

I begin by articulating some of the concerns expressed by bioethicists about reproduction in the context of emerging technologies. I then argue that although Mertes and Pennings' account of reproduction is adequate to address these concerns, it is unnecessarily restrictive. In Section 2, I examine James Griesemer's account as an alternative to Mertes and Pennings'. I believe Griesemer's account, although motivated by problems that arise in evolutionary theory, does a better job capturing the parent-offspring relation in the context of emerging technologies. Even so, his account isn't without problems. While it isn't too restrictive, which is my complaint against Mertes and Pennings, Griesemer's view is overly permissive. Too many things end up qualifying as parents. In an effort to find the right balance, I propose a new account of reproduction, which is neither too permissive nor too narrow, called the Overlap, Development and Persistence (ODP) account. In the final section, I argue that even though the ODP account doesn't mention genes, it best captures the meaning of 'genetic parenthood'.

\section{Reproduction and the Family Tree}

Nuclear families are increasingly composed of individuals sharing sociological rather than genetic ties, but it would be a mistake to assume that this means genetic ties have lost their importance: fertility services are a growing market in a world in need of adoptive parents, and genetic parents continue to be afforded legal rights and responsibilities (whether they want them or not). In addition, a growing number of states grant adopted children the legal right to know their birth parents in order to access valuable information about their medical histories and historical identities. Another sign that the value of genetic ties remains steady is the increasing 
number of ways available to 'assist' reproduction, e.g. ovulation induction, intrauterine insemination, in vitro fertilization, egg donation, sperm donation, gestational surrogates, oocyte cryopreservation and uterine transplantation. In fact, researchers have gone beyond merely 'assisting' reproduction by inventing new ways to become a genetic parent. The reprogramming of adult skin cells into sperm and egg cells might, for example, provide a way for same-sex couples to have their own children (Ringler 2015). And, as a final example, reproductive cloning may provide the option of becoming the ultimate single-parent (Kass \& Wilson 1998). So whether assisted or invented, the ability to have children that are 'one's own' really matters to a lot of people. ${ }^{3}$

Some bioethicists have begun to wonder, however, whether these new methods of becoming a genetic parent are actually reproductive in kind (see, for example, Alpern 1992; Douglas 2014; Kolers 2003; Kolers \& Bayne 2001, 2003; Mertes 2014; Mertes and Pennings 2008; Silver and Silver 1998; Sparrow 2006, 2012). As Mertes and Pennings explain:

$[\mathrm{T}]$ he prospect of producing gametes derived from customized embryonic stem cells [to help same-sex couples have their own children] presents such an enormous "detour" from natural reproduction that some inquiry is warranted into the question of whether it can deliver what it promises, namely, genetic parenthood. (2008: 13)

Given the 'enormous detour' of producing gametes from customized stem cells, we might wonder what it would take to deliver on the promise of genetic parenthood. What must the

\footnotetext{
${ }^{3}$ Of course, whether genetic ties ought to hold the kind of importance people place on them is a separate question. For more on that topic, see, Brake \& Millum, 2016.
} 
process of using gametes derived from stem cells look like for the children coming out at the other end of that process to qualify as 'one's own'?

One way to think about an answer to this question is by way of paternity and maternity tests. These tests take genetic similarity as evidence of a parent-offspring relation and are designed to inform an individual of whether the child in question is 'one's own'. But making an inference from genetic similarity to genetic relation can be deeply misleading. For example, I share half of my genes with my mother, but I also share half of my genes with my sister, half with my brother, and half with my offspring. Whether I am another individual's mother, sister or brother can't be determined by looking merely at genetic similarity. Moreover, if my father happens to be an identical twin, even the most sophisticated paternity test will identify his brother as my father, since there will be an overwhelming genetic similarity between them. Jacqueline Laing (2006) uses this last example to show how a genetic paternity test can produce a false match leading to disappointing results:

Imagine an adult adopted as a child who is seeking out his father. Suppose he discovers that there is a match for paternity with $\mathrm{X}$. He is elated but soon discovers that $\mathrm{X}$ is not his father but the twin of his father, $\mathrm{Y}$. The discovery that $\mathrm{X}$ is not his father at all, but his uncle, will be a matter of great significance even though the DNA for both $\mathrm{X}$ and $\mathrm{Y}$ might be the same. (551-2)

Liang argues that even though the match is genetically identical to the adoptee's father, in the eyes of the adoptee the search is a failure. He did not find his father, merely someone who is genetically identical to him. Again, genetic similarity doesn't determine the nature of genetic 
relations. Kenneth Alpern (1992) makes the same point with a different example. Imagine that while walking down the street, you discover that a baby happens 'to have exactly the same genetic makeup as you'. Alpern then asks, 'Would this make the child yours or you its parent?' The answer seems to be no. To have the same pattern of genes 'surely is not enough to constitute having one's child' (160).

While these thought experiments aren't enough to tell us why genetic relations matter to intending parents, they do tell us something important about those relations: that an individual shares genes with another tells us almost nothing about the nature of their genetic relation. A child is not 'one's own' merely in virtue of sharing one's genes. But as evident as that may be, it's easy to fall into the mistaken assumption that similarity indicates genetic relation. The tendency is especially apparent in discussions of human cloning where individuals who openly decry the mistake go on to make it. Consider two different passages from Robert Sparrow (2006): in the first, he shows the mistake; in the second, he commits it. According to Sparrow, the 'informational metaphor' encourages us to mistakenly measure relations between individuals by comparing their genetic blueprints. He writes:

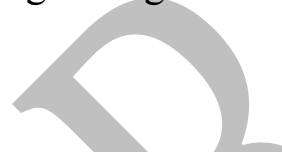

The problem with the informational metaphor is that it ignores the role played by history in determining even our sense of genetic relatedness. What is missing in these cases is the appropriate causal connection between the genetic make-up of the parties involved. Our genetic relation to others is not merely a question of the genes we happen to share, but also a question of the history of how we came to share those genes. (314) 
Above, Sparrow is clear that we cannot discover who is genetically related to whom merely by looking at genetic similarity.

In a different passage, however, Sparrow argues that clones share too many genes with their donors to be considered their children. Consequently, an individual who clones herself would have less of a parental claim to her clone than her (the donor's) own parents. Sparrow writes:

If it is genetic relatedness that grounds a claim to parenthood then they [the parents of the DNA donor] would surely have a better claim than the DNA donor. Any suggestion that the DNA donor should be granted custody of the clone against their own parents, on the grounds that they (the donor) are 'more related' to the clone, risks the ludicrous conclusion that I am more the parent of my identical twin than are my own parents. (315)

According to Sparrow, the DNA donor is unlikely to qualify as the clone's parent because she shares too many genes with the clone. Instead, the parents of the DNA donor, who share the right amount of genes with the clone, ought to qualify as its parents. This is an argument that depends on genetic similarity. So even though Sparrow is against using genetic similarity as an indication of genetic relatedness, he ends up relying on it to guide his intuitions when constructing an argument about the relation of a clone to its donor.

Sparrow is not alone in this. A number of scholars have relied on genetic similarity to conclude that a clone does not qualify as a child of the DNA donor but, rather, as the donor's delayed twin (see, for example, Lewontin 1998; Silver \& Silver 1998). Of course, the problem 
with such claims is not in making them or with their intuitive basis. Instead, the problem is that such claims rest merely on intuition and lack any principled justification. As I've already established, and as authors such as Sparrow admit, genetic similarity by itself is not sufficient to determine the genetic relation between individuals, which means that the genetic similarity between a DNA donor and her clone is not sufficient to justify the claim that the two share the same parents. To resolve this problem, and to provide the justificatory basis for the resolution, we need to look at the process that gives rise to the genetic similarity between the DNA donor and her clone. Doing so, will help us determine whether the process is an instance of reproduction. If it is, the clone may very well be the child of the DNA donor. If it isn't, we'll have provided the needed justification for Sparrow's intuitions. Let's turn, then, to views about the nature of reproduction.

\subsection{The Concept of Reproduction in Bioethics}

Several bioethicists have attempted to capture features of the reproductive process by examining the genetic parent-offspring relation. In addition to Sparrow, who emphasizes the need for an 'appropriate causal connection' between parent and child, Avery Kolers (2003) has argued that individuals stand in a parent-offspring relation only when the genes they share have been physically transmitted from parent to child. According to Kolers, reproduction is an 'embodied process' that requires genetic information to be 'organized into chromosomes, on

strands of DNA, built from environmental raw materials, [and] subject to expression or mutation...' (406). To ignore this physical aspect of genetic transmission (and the reproductive process) is to view genes as a type of entity that 'is individuated in terms of the information it carries' (406). Construed this way, genes would be carriers of information in something like the 
way sentences, knowledge states, or beliefs carry information. But the causal connection between parent and child doesn't make sense when genes are construed this way, since that causal connection is formed by the transmission of concrete objects, viz., genes. If this is right, then tracking the shared genetic information of two individuals won't be a matter of looking for a type of information transmission, but rather, a concrete token of that transmission. Notice, then, that Kolers's type/token distinction helps to explain the intuition that finding a random baby who shares one's genetic sequence doesn't justify the inference that the baby is 'one's own'. In order to qualify as one's own, the child must receive one's gene tokens. It's not enough for the baby to have the same type of genetic sequence as oneself.

Building on Sparrow's account, which emphasizes causal connection, and Kolers's, which emphasizes physical transmission, Mertes and Pennings add another component to the process of reproduction, viz., reshuffling. From the observation that mixing two genomes into one is a basic feature of human reproduction (and sexual reproduction more broadly), they argue that the 'physical genes have to be reshuffled once while going from parent to child' (2008: 13) for the process of genetic transmission to count as an instance of reproduction. Their account was one of the first to provide an explanation for the intuition that a DNA donor does not count as a clone's parent. The idea is that although physical genes are transmitted from a DNA donor to a clone in a way that amounts to a causal connection, the genes are not reshuffled in the process of that causal interaction. Instead, both the clone and its donor share the same DNA sequence, which is the product of a reshuffling event initiated by the DNA donor's parents. As a result, the clone is the DNA donor's delayed twin. This is what many scholars have assumed, albeit without justification. Whether the DNA donor's parents know it or want it, they now have another child: a younger, identical twin to the child they've already produced. 
As this example demonstrates, Mertes and Pennings' account helps to identify a child's genetic parents, even when the reproductive process that brings about the child (in this case a clone) is unfamiliar. But a major weakness of their account is that it is too narrow. If we assume that 'genetic parenthood' and 'reproduction' are two concepts that travel together, then Mertes and Pennings' account of genetic parenthood implies that most organisms on earth do not reproduce. After all, asexual reproduction is much more common than sexual reproduction and doesn't involve reshuffling. Providing an account of a concept like reproduction that ends up excluding most of its instances seems like a mistake. But in their defense, Mertes and Pennings were likely motivated to provide an account of specifically human reproduction, since it's assisted reproductive technologies that give rise to puzzles about genetic parenthood. Even so, there are two critical problems with their choice to constrain reproduction to cases of sexual reproduction.

First, although humans have always reproduced sexually, sexual reproduction is not an essential feature of our species. This is one lesson of our evolutionary history. Species do not have essences, they are historical individuals (Ghiselin 1974; Hull 1978), which means that any feature we take to be essential to a species can change over time. That's not to say that anything may count as reproduction or that the reproductive process can undergo any change. It is possible for a process to cease to fall under the scope of the concept, even if saying how and when requires more clarity about the concept. We can also allow quite a bit of flexibility in how we understand the concept of reproduction, as is evident from the difference between asexual and sexual reproduction. Asexual reproduction involves the transmission of all genes, but sexual reproduction involves transmission of only half. But we can set these points to the side to take note of what is important here: when trying to understand what it means for humans to 
reproduce in an age where that is an open question, it is arbitrary and question begging to constrain the answers using requirements that aren't essential to the species. Sexual reproduction, and the reshuffling of genetic sequences involved in it, may be typical of our species, but it isn't essential.

In response, Mertes and Pennings might say that they are merely providing a description of human reproduction and not an account of what should count as human reproduction. This leads to my second complaint about their choice to constrain reproduction to the human variety. If it were possible for Mertes and Pennings to avoid my first complaint by insisting that they are engaged in a merely descriptive project, there would be little reason to emphasize reshuffling as an essential feature of reproduction. After all, reshuffling is only one part of sexual reproduction. Why not include other features of the reproductive process that seem just as important, e.g. reduction from diploid to haploid number of chromosomes during meiosis, restoring the diploid number during fertilization, etc.? It's because their account is not merely descriptive. By choosing some aspects of sexual reproduction and ignoring others, Mertes and Pennings offer an account of what they take to be particularly important to the process of human reproduction. This shouldn't be surprising. They're interested in capturing the meaning of genetic parenthood, so it's natural to anticipate the importation of value judgments into the proposed solution. But in this case, emphasizing the value of reshuffling is misdirected, since it ends up guiding Mertes and Pennings to an overly narrow notion of reproduction. And, anyway, for all that has been shown, it may be possible for human beings to reproduce without the reshuffling Mertes and Pennings emphasize. To assume otherwise is question begging. Even so, reshuffling does play an important role in human reproduction, and a more general account of reproduction should make room for it, even if it doesn't require reshuffling to play that role. What, then, is that role? 
Answering this question will help us arrive at a broader account of reproduction, one not constrained by anthropocentric intuitions.

\subsection{The Role of Reshuffling in Reproduction}

To understand the role of reshuffling in reproduction, let us first make clear what the process of reproduction promises to deliver. One strong motivation for reproducing rather than adopting a child is the expectation that a child of 'one's own' will resemble its parents. Reproduction, then, promises to deliver what adoption can't: a resemblance based on shared genetics. Indeed, it's in virtue of the tie between phenotypic resemblance and genetic similarity that we're mistakenly tempted to conclude that the latter is indicative of a parent-offspring relation (and why we need thought experiments to show us why that isn't always the case). Of course, parents and children don't always end up resembling each other, but the expectation remains legitimate. And even if offspring don't always resemble their parents, the chance that they will is much higher than between two individuals chosen at random. This is why Charles Darwin used the expected resemblance of parents and offspring in his theory of evolution by natural selection, why Gregor Mendel predicted the expected resemblance between parents and children in his pea experiments, and eventually, why Rosalind Franklin, Francis Crick and James Watson expected to find its material basis in their discovery of DNA's structure.

Although Franklin, Crick, and Watson expected the discovery of DNA's structure to show how information gets encoded and passed from parent to offspring, they also expected that discovery to explain the basis of parent-offspring resemblance. It turns out, however, that merely transferring information encoded in DNA is not sufficient to explain resemblance. There are a host of presuppositions built into the transference of DNA material, and if any of those 
presuppositions go unmet, parent-offspring resemblance may be undermined. Even something as basic as the cellular environment must be transferred along with DNA in order for information encoded in genetic material to be properly expressed. And the environmental factors essential to gene expression may extend far beyond the inside of a cell.

When thinking about the role played by reshuffling in reproduction, it's important to keep in mind that it is resemblance that matters, not identity. Offspring cannot be identical to their parents if a biological population is to evolve by natural selection. When Darwin formulated his theory, he noticed that for things to evolve there had to be variation in the products of reproductive processes. He observed that there was variation in any population of organisms and he knew that some individuals have more offspring than others, so he reasoned that if offspring inherited some of that variation through reproduction, the composition of the population would change over time. Only in this way could a population evolve by natural selection - this turns out to be true for populations of cells, genes, and organisms. Continued reproduction requires there to be a source of variation, which allows for the composition of a population to change in a way that is favorable to a new environment. Without variation in resemblance over time, groups of organisms die.

But what mechanisms allow successive generations to resemble each other in nonrandom ways, while, at the same time allowing for the kind of phenotypic variation necessary for natural selection? Combining Sparrow's, Kolers's, and Mertes and Pennings' reproductive requirements provides an answer in the case of human beings: the physical transmission of genes produces offspring with more than random resemblance to their parents and the reshuffling of genes enables offspring to vary from their parents. Thus, we have arrived at an answer to our previous question: What is the role of reshuffling in human reproduction? It accounts for 
novelty: the reshuffling of genes creates an opportunity for offspring to acquire traits different from their parents. But reshuffling isn't the only way for human beings to get the necessary variation in traits. And if that's right, it would be a mistake to build an account of human reproduction that insists on emphasizing reshuffling's role in that process. Indeed, a more permissive account of reproduction may better explain both resemblance and novelty without relying on reshuffling.

\section{Reproduction and the Tree of Life}

So far we have looked at reproduction as a way of extending one's family tree, but reproduction also extends a much bigger tree: the tree of life. Not surprisingly, researchers interested in tracking changes in biological populations over time as well as the evolution of new species, also have an interest in a clear concept of reproduction. ${ }^{4}$ After all, they must decide which organisms belong to one generation and which to the next, whether two seemingly different organisms are actually just one organism, whether one individual is particularly good at breeding, which organisms are siblings, and so on. All of these tasks rely on some concept of reproduction, and unlike the bioethicists looking for a concept of reproduction applicable to new reproductive technologies, researchers interested in reconstructing the tree of life are not constrained by such narrow aims. Instead, they want to know what is necessary for any organism to reproduce in a manner that would satisfy the dual requirements of resemblance and novelty. That is, they want an account of reproduction that fits within the framework of evolution by natural selection. Whatever that account is, it should also allow for the reproductive process

\footnotetext{
${ }^{4}$ Philosophers of biology who have written about reproduction and its role in natural selection include Bapteste \& Dupre 2013; Booth 2014; Bouchard 2008; Clarke 2011; Dupre \& O’Malley 2007; Ereshefsky \& Pedroso 2013; Godfrey-Smith 2009, 2016; Griesemer 2000a, 2000b, 2000c; Hamilton and Haber 2006; Jablonka 2002; Szathmary \& Maynard Smith 1997; Okasha 2006; O’Malley 2016.
} 
itself to evolve over time. An account of reproduction, then, should 1) make room for resemblance, 2) allow novelty, and 3) be malleable. ${ }^{5}$

With those goals in mind, Griesemer (2000a, 2000b, 2000c) has put forward a view of reproduction that I believe is better suited to accommodate both human and non-human varieties. If I'm right, we can use it as a principled basis for thinking about emerging reproductive technologies. His account has two parts. First, Griesemer requires that some of the parts of the offspring were once parts of the parents. This requirement, which he calls 'material overlap', separates reproduction from other processes, like, for example, copying. A photocopier produces identical copies, and in that sense reproduces the original, but since there is no material overlap between the original and its copies, the latter are not offspring of the former according to Griesemer. The faithful transmission of properties from one entity to another is not necessary for reproduction, 'Parents can be non-winged while offspring are winged. Parents can be asexual while offspring are sexual. And so on' (Griesemer 2000a: S362-3). We wouldn't want the duplication characteristic of photocopying included in an account of biological reproduction, anyway, since exact duplication is not conducive to evolution by natural selection. Instead, what we want is to preserve the genealogical relation characteristic of reproduction while maintaining our dual commitment to generational resemblance and novelty. Requiring material overlap can accomplish this. As he writes, 'Material overlap is the relation that results from the flow of matter which creates genealogy. In politics you follow the money, in biology you follow the stuff' (Griesemer 2000a: S359).

\footnotetext{
${ }^{5}$ An account of reproduction should be malleable because the process of reproduction may itself evolve over time. It was once the case that all organisms reproduced asexually, then, through evolution, organisms started reproducing both sexually and asexually. Evolution may have further, unanticipated tricks up her sleeve and a general account of reproduction should be malleable enough to accommodate them.
} 
But as Griesemer notices, merely requiring material overlap is not sufficient for an account of reproduction, since alone it generates counterintuitive results. For example, if instead of putting the paper into a photocopier one were to cut it in half, the 'material overlap' between the original piece of paper and the part that has been cut off would satisfy Griesemer's first requirement. Part of the 'parent' is now part of the 'offspring' and yet it would be strange to say that the paper has thereby reproduced. Griesemer's second requirement is meant to block this odd conclusion. In addition to material overlap, the process of biological reproduction requires that the parent confer on the offspring the capacity to develop into something capable of reproduction. ${ }^{6}$ This additional requirement is meant to make sense of the obvious fact that living things continue to reproduce indefinitely. With each generation of reproducing individuals, the offspring inherit material parts from their parents that allow them to develop their own reproductive capacities. ${ }^{7}$

Griesemer emphasizes the fact that the capacity to reproduce is not something that is transferred directly, but develops in successive generations of individuals. He writes:

Typically, the capacity to reproduce must be acquired or built-up; things are not born with it. Even cleavage cells in an early embryo that do not take up nutrients or synthesize new RNA must go through some internal rearrangement in order to successfully divide again. At the very least, they must move chromosomes to the

\footnotetext{
${ }^{6}$ This criterion carries implications for individuals with infertile children. Does this criterion mean that the parents of infertile children haven't in fact reproduced? Yes. I return to this issue further on in the paper.

${ }^{7}$ It is unfortunate that Griesemer uses the word 'reproduction' in the second requirement because it makes his account appear circular, i.e. in order to reproduce, one must develop the capacity to reproduce. Griesemer seems aware of this problem when he emphasizes that his account is 'recursive', not 'circular' (2000a: S361). Indeed, the circularity seems to go away if we rephrase the second requirement without using the word 'reproduction.' For example, we can describe both of Griesemer's requirements as follows: material parts of parents become parts of offspring and these parts confer the developmental capacity on offspring to restart the process of material transfer.
} 
metaphase plate and pull them apart in order to divide in a way that yields a reproductively capable offspring cell. (2000b: 246)

This developmental feature of the capacity to reproduce, which is acquired by an organism's offspring via material overlap, helps to account for the dual requirements of variation and resemblance. Indeed, the fact that the capacity to reproduce develops from materially transferred parts increases the likelihood that offspring will resemble their parents. Since shared material parts are more likely to give rise to offspring with features that resemble the features of their parents than those of randomly selected individuals, "realization in offspring of the capacity to reproduce will undoubtedly entail many particular trait resemblances' (Griesemer 2000a: S361). But Griesemer's requirements also leave room for generational novelty. Given that the capacity to reproduce is not simply copied from parent to offspring, but must develop, variations are likely to arise in the process of development. As Griesemer explains:

If heredity were exact, then evolution would come to a halt...heredity is likely to be exact whenever development is null. Variation "emerges" in the developmental process of acquiring the capacity to reproduce. Null developers need not acquire reproductive capacity, so the opportunity for variation which nevertheless leads to reproductive capacity is absent. (2000c: 74$)$

In summary, the process of acquiring the capacity to reproduce, through the development of materially transferred parts, gives rise to the novelty and resemblance we expect to find between 
parents and offspring. Thus, Griesemer's account can fulfill the requirements of reproduction without reshuffling, offering a more permissive alternative to Mertes and Pennings' account.

It is also worth noting a further virtue of Griesemer's account. Since his account of reproduction requires new generations to acquire the capacity to reproduce, it avoids potential problems with 'mere bodily byproducts' and clear cases of transplantation. Bodily byproducts, e.g. hair clippings, meet the first criterion of material overlap, but not the second. Hair clippings used to be a material part of an individual, but they lack the ability to develop into something capable of reproduction. Hence, on Griesemer's account, one does not reproduce in virtue of cutting one's hair. The same goes for most organ transplants, e.g. kidney transplants. There is material overlap between the recipient and her kidney donor, but she does not develop a capacity to reproduce in virtue of the donation. Even transplants of reproductive organs, such as a uterine transplant, do not satisfy both of Griesemer's requirements. A transplanted uterus enables one to reproduce, but it does not confer upon the recipient the capacity to develop the reproductive capacity, since a transplanted uterus is already developed. Thus, most organ transplants, including reproductive ones, fail to meet Griesemer's second requirement when properly understood.

\section{A New Concept of Reproduction}

We have now looked at the concept of reproduction from two perspectives. A number of bioethicists are interested in developing a concept of reproduction that can resolve questions of genetic parenthood in cases of assisted reproduction. To this aim, Mertes and Pennings provide an account of reproduction that relies on familiar features of human reproduction in order to articulate conditions they believe to be sufficient for a process to qualify as an instance of 
reproduction. In contrast, those working in the biological sciences want a concept of reproduction that can help track the parent-offspring relations needed to reconstruct the tree of life. Guided by this aim, Griesemer identifies conditions that seem to be necessary for reproduction, and articulates a conception that fits nicely with our understanding of evolutionary theory.

Despite their different aims and the discrepancies between them, there remain significant similarities between the two accounts. For instance, they both take reproduction to be a causal process that requires the transference of physical objects, they both articulate a concept that accounts for novelty in the products of reproduction, and they both aim to account for resemblance between parent and offspring. Of course, Mertes and Pennings argue that the physical objects to be transferred must be genes, which serve to produce the necessary resemblance relations as well as novelty through their reshuffling. Griesemer's view is more general and is committed only to the more generic view that material overlap be present in cases of reproduction. This material overlap gives rise to both resemblance and novelty as the result of developing a reproductive capacity.

So, although the main ideas are the same (this shouldn't be surprising given the fact that we're after an account of reproduction), the details of each approach are different enough to generate divergent answers to questions of biological parenthood in cases of third-party reproduction. To see this, consider again the case of mitochondrial DNA donation. On the account offered by Mertes and Pennings, the donor of mitochondrial DNA does not qualify as a third genetic parent, since the DNA is not reshuffled in the process of donation. On Griesemer's account, however, we're likely to get the opposite conclusion. Although there is clearly material overlap between the donor and the child, it's uncertain whether mitochondrial DNA (mtDNA) 
confers on a child the ability to develop his or her own reproductive capacity. If it does, the donor will qualify as a third parent; if not, then not. But if we consider the fact that mtDNA acts as a cellular energy source, it probably helps the entire organism develop into something with its own reproductive capacity (see, for example, Guantes, Diaz-Culunga and Iborra 2016). Consequently, the donor of mtDNA will likely qualify as a third parent.

Notice, however, that Griesemer's account does not provide a straightforward answer to the problem raised by mtDNA donation. This is potentially a big problem when using his account of reproduction to think through problems that may arise for cases of assisted reproduction. Since Griesemer never explains how, or to what extent, transferred parts (the ones that create the material overlap) must be involved with the development of the recipient's reproductive capacity (recall that this is Griesemer's second requirement), there is the potential that the transference of parts not typically associated with a reproductive process would nevertheless qualify as an instance of reproduction. For example, suppose I were to receive a stem cell transplant designed to reconstruct a severely damaged uterus (instead of, say, receiving an entirely new transplanted uterus). Well, given that supposition, on the account offered by Griesemer, the donor of the stem cell material could qualify as my parent. After all, the transfer involved material overlap and it conferred on me (the recipient) a capacity to develop my own reproductive capacity. But that seems wrong.

If Griesemer's account turns a stem cell donor into a biological parent, we might worry that it is overly permissive. But as we've seen, Mertes and Pennings' account is overly restrictive, since it excludes instances of reproduction that do not involve the reshuffling of genes. Either way, we need something better, an account of reproduction that is neither too restrictive nor too permissive. My solution is to add a third requirement to Griesemer's account, 
which will save it from being overly permissive. Recall that Griesemer's first requirement is that there be material overlap between parent and offspring; the second is that the inherited parts contribute to the development of the offspring's own reproductive capacities; my proposed third requirement is that the material parts, or their descendants, be passed down to the next generation. Only when these three conditions are met will the initial material transfer count as an instance of reproduction. If the parts that create the material overlap and develop the recipient's capacity to reproduce die with the recipient, and are not passed down to the next generation, the initial transfer doesn't count as reproduction. If we label Griesmer's requirements 'Overlap and Development' and add my third requirement, 'Persistence', we end up with my proposed account: Overlap, Development and Persistence (ODP).

I believe my third requirement captures what Griesemer probably assumed to be a natural consequence of meeting his overlap and development requirements. But that's a significant assumption. So why would Griesemer assume that some of the parts of the parent that helped the offspring develop its own capacity to reproduce would get passed down to the next generation? The answer is obvious: Griesemer's engagement with the concept 'reproduction' is done in a context free of human-invented ways of merging parts, e.g. stem-cell transplants. Instead, the context of his engagement with 'reproduction' is as a process characteristic of biological propagation, which is a process that has existed for a long time, well before humans ever existed, and involves the creation of a new organism from the parts of another. As the product of such a process, a new organism, along with its own capacity to reproduce, develops naturally from parts it has inherited. Since the new being is made from the physical parts of its parent, its own parts will inevitably get passed down to make the next generation, and that future generation will contain parts of its parent (or descendants of the parts of its parent). After all, the third 
generation is also made from those parts (or their descendants). It is this overlap of parts between generations that creates a lineage.

Griesemer most likely assumed that if transferred parts are involved in the development of the recipient's capacity to reproduce, the transmission of those parts to the next generation is inevitable. This is certainly the case for the vast majority of biological processes that qualify as reproduction. Where transferred parts give rise to an entire organism, they also end up being passed down to create the next generation, and so on. Given the development of biological technology and our ability to merge biological parts in new and inventive ways, however, this assumption isn't always unproblematic. A stem cell transplant aimed at reconstructing a uterus in its recipient appears to meet Griesemer's requirements - through material overlap, the transferred parts confer on the recipient the capacity to develop its own reproductive capacityand qualify as an instance of reproduction. On the ODP account, however, stem-cell transfers aimed at restoring an individual's reproductive capacities will not give rise to a parent-offspring relation, because neither the transferred parts nor their descendants will be passed down to the next generation. The stem cells and their descendants die with the recipient in the same way that the cells comprising a kidney transplant die with its recipient. Thus, the additional requirement I propose allows us to remain true to the spirit of Griesemer's account while avoiding the objectionable feature that makes his account too permissive.

I want to make one last point about the persistence requirement, a point readers are likely to find controversial. If the requirement demands that parts persist to the next generation, what happens if my offspring fails to reproduce? The first two requirements are met—some of my parts are transferred and develop in the recipient its own capacity to reproduce- - but the third requirement remains unfulfilled. The recipient of my parts does not itself reproduce. 
Consequently, it appears that I have failed to reproduce, despite the fact that I have a child. So, do I really want to insist on my third requirement? Yes. The worry is allayed if my third requirement is understood counterfactually: it's not necessary that the parts contributed by me, which play a role in the development of my offspring's ability to reproduce, actually persist to the next generation. Instead, it's enough that those parts be of a type that would be passed down if, contrary to fact, I were to reproduce.

But softening the requirement in this way doesn't avoid another potential problem. Has an organism failed to reproduce if its offspring is itself incapable of reproducing? Is producing sterile offspring failed reproduction? In answer, consider two examples of organisms that never develop the capacity to reproduce: worker bees and mules. In each case, the parents produce offspring that never develop the capacity to reproduce. So, yes, these are instances of failed reproduction. A queen bee has not reproduced when she produces worker bees and a horse and donkey have not reproduced when they produce a mule. But this conclusion is entailed by Griesemer's account, too. As he notes, 'The production of offspring that do not have the capacity to reproduce is not reproduction' (2000b: 246). A potentially controversial conclusion, perhaps, but it's one consistent with the recursive nature of reproduction: offspring inherit material parts from their parents that allow them to develop their own reproductive capacities. Failure to meet that condition is not reproduction.

\section{ODP: An Account of Reproduction Suitable for Genetic Parenthood}

As we've seen, the phrase 'having a child of one's own' has become synonymous with becoming a genetic parent. But what's the value that genes track? Why is it so important that a child have one's genes? One difficulty in answering these question lies in the fact that 'gene' is 
an ambiguous concept, and some specifications of it will be better at picking out the value implied by the genetic parent-offspring relation than others (Rheinberger, Müller-Wille, and Meunier 2015). In the final section, I want to address this ambiguity to show that the ODP account — which does not mention genes — can best handle difficulties associated with 'genetic' parenthood.

What, then, are the potential meanings of the word 'gene'? One way to understand 'genes' is as the drivers of development, or, differently, as the things that specify the phenotype (Bromham 2016). This is a natural interpretation of the idea, since genes provide something like a recipe for how an organism will turn out—its phenotypic features. But this interpretation isn't without problems. If we take the significance of 'genes' in 'genetic parenthood' to be their ability to specify the phenotype, we'll have to decide whether other things that contribute to specifying the phenotype (e.g. environmental factors) should also count as parental contributors. This is a potential problem in cases of 'collaborative reproduction', where the environment provided by a surrogate influences the specification of a child's phenotypic traits (Perera \& Herbstman 2011). Indeed, Charis Thompson (2005) has argued that multiple people can come together and contribute 'horizontally' or 'laterally' to the creation of a child and qualify as parents in virtue of that contribution. As she has written, 'Assisted reproductive technologies have introduced a collaborate reproduction that involves a gamete donor, an embryo donor, or a surrogate, thereby lateralizing "descent" (12). But even if this sense of 'gene' can be a marker for relations that may be inherently valuable in ways similar to the value of a parent-offspring relation, should a surrogate count as the child's genetic parent? Not if we want to prevent the concept of genetic parenthood from becoming nearly vacuous. After all, many environmental factors that are straightforwardly not genetic parents, e.g. a child's climate, nutrition, her 
daycare, the air quality of the city in which she lives, water quality, etc., causally contribute to the development of a child's phenotype. Hence, understanding genes as things that specify the phenotype seems to allow for 'too many parents'.

My ODP account helps avoid this problem. If genes are important as trackers of the value inherent in parent-offspring relations, it is because they are the physical things that get transferred from parent to child. Without that material overlap, something that may contribute to the development of a child's phenotypic traits may remain valuable, but it won't be valuable in the same way - that is, as a marker of genetic parenthood. A gestational surrogate is clearly an important biological contributor to a child's creation, but if none of her parts become parts of the child she is carrying, she is not the child's genetic parent on the ODP account. ${ }^{8}$ The same point applies to other environmental components that obviously contribute to the development of a child but are just as obviously not genetic parents. Thus, the material overlap requirement prevents the concept of genetic parenthood from becoming nearly vacuous.

If understanding genes as things that specify the phenotype permits too many things to qualify as genetic parents, a more promising path might be to understand 'gene' as a historical document. On this understanding, tracking genetic parent-offspring relations would consist of tracking a genetic historical record without concern for phenotypic consequences. For example, one might track parts of the genome called 'microsatellites', which make no difference to phenotypic development but can be used to trace an individual's ancestry. Notice, however, that in contrast to 'genes' as phenotypic contributors, this sense of 'gene' requires that they be faithfully transmitted. And understood this way, the value inherent in ancestor-descendant

\footnotetext{
${ }^{8}$ That's not to say that a gestational surrogate can never be a genetic parent to the child she is carrying. Occasionally, some DNA from the surrogate can pass through the placenta and become part of the fetus. If this were to happen and the transferred DNA were to fulfill all three requirements of the ODP account (an unlikely scenario), then the surrogate would qualify as the child's genetic parent.
} 
relations is traced by what is invariant between generations of individuals. Recall, however, that parents contribute parts that develop the offspring's capacity to reproduce in order to account for both resemblance and novelty in genealogical relations. In the process of developing a capacity to reproduce using the material parts (or their descendants) of the parent, a child is likely to both resemble and vary from its parents. But if we understand 'genes' as historical documents, ignoring phenotypic consequences, we end up relying on a view that fails to account for the expected phenotypic resemblance and novelty of genetic parents and children. Understood as an historical record, then, the idea of a gene won't be able to do what we want it to do. The ODP account avoids this problem.

What we see, then, is that my proposed account best captures the value of the genetic parent-offspring relation. It avoids the problem of counting everything that contributes to phenotype as a genetic parent, which is the case for gene concepts that understand 'genes' as those things that contribute to phenotype. My account also avoids problems associated with understanding 'genes' as historical documents, a view that ignores phenotypic consequences. Instead of these two problematic views of the 'gene' concept, what we need if we want to track what seems inherently important in the genetic parent-offspring relation is what is offered by the ODP account: we have a genetic parent-offspring relation when what is transmitted from one generation to the next involves material overlap, contributes to the phenotype of the offspring, and persists to the future generation. Indeed, I believe this notion of a 'gene' best tracks what we find valuable about 'genetic parenthood'.

Let me conclude by briefly summarizing what I have argued. With the increasing number of ways available to 'assist' reproduction, some bioethicists have started to wonder what it takes to deliver on the promise of genetic parenthood. That a child shares one's genes is not enough to 
make it 'one's own'. Instead, we must look at the process which gives rise to genetic similarity to determine whether it is reproductive in kind. Mertes and Pennings have offered an account of reproduction meant to capture this process and help discriminate between genetic parents and nonparents. However, their account requires gene reshuffling, making it unnecessarily restrictive. I have offered an alternative to Mertes and Pennings, using Griesemer's account of reproduction. Although Griesemer's account does a better job of capturing the genetic parentoffspring relation, his account turns out to be overly permissive. In an effort to find the right balance, I have proposed the Overlap, Development and Persistence (ODP) account of reproduction. Finally, I have argued that even though the ODP account doesn't mention genes, it best captures the meaning of 'genetic parenthood'?

\footnotetext{
${ }^{9}$ Special thanks to Sean Allen-Hermanson, Ellen Clarke, Avery Kolers, Tim Lewens, P. D. Magnus, Matt Mosdell, Bonnie Steinbock, and anonymous reviewers for their careful reading and constructive criticism.
} 


\section{References}

Alpern, K. D. (1992) 'Genetic Puzzles and Stork Stories', in K. D. Alpern (ed.) The Ethics of Reproductive Technology, 147-169. Oxford: OUP.

Bapteste, E. and J. Dupre (2013) 'Towards a Processual Microbial Ontology', Biology and Philosophy, 28/2: 379-404.

Booth, A. (2014) 'Symbiosis, Selection, and Individuality', Biology and Philosophy, 29/5: 657673.

Bouchard, F. (2008) 'Causal Processes, Fitness, and the Differential Persistence of Lineages', Philosophy of Science 75/5: 560-70.

Brake, E. and J. Millum (Fall 2016 Edition) 'Parenthood and Procreation', in E. N. Zalta (ed.) The Stanford Encyclopedia of Philosophy, Available at: $<$ http://plato.stanford.edu/archives/fall2016/entries/parenthood/>

Bromham, L. (2016) 'What is a gene for?', Biology and Philosophy 31/1: 103-123.

Clarke, E. (2011) 'Plant individuality and multilevel selection theory', in K. Sterelny and B. Calcott (eds.) The Major Transitions Revisited, 227-250. MIT Press.

Douglas, T. (2014) 'Stem cell-derived gametes, iterated in vitro reproduction, and genetic parenthood', Journal of Medical Ethics 40: 723-4.

Dupre, J. and M. A. O’Malley (2007) 'Metagenomics and biological ontology', Studies in History and Philosophy of Science Part C 38/4: 834-846.

Edwards, J. (2014) 'Undoing Kinship', in T. Freeman, S. Graham, F. Ebtehaj, and R. Martin (eds.) Relatedness in Assisted Reproduction, 44-60. Cambridge, MA: Cambridge University Press.

Ereshefsky, M. and M. Pedroso (2013) 'Biological Individuality: The Case of Biofilms', Biology and Philosophy 28/2: 331-349.

The Human Fertilisation and Embryology Authority (2015) 'Mitochondrial donation: Clinic staff guide to consent', Version 1: 1-27. Available at: <http://www.hfea.gov.uk/9939.html > accessed 24 July 2015.

Ghiselin, M. T. (1974) 'A Radical Solution to the Species Problem', Systematic Zoology 23/4: 536-544.

Godfrey-Smith, P. (2009) Darwinian Populations And Natural Selection. Oxford: OUP.

Godfrey-Smith, P. (2016) 'Complex Life Cycles and the Evolution Process', Philosophy of Science 83/5: 816-827.

Griesemer, J. (2000a) 'Development, Culture, and the Units of Inheritance', Philosophy of Science 67 (Supplement. Proceedings of the 1998 Biennial Meetings of the Philosophy of Science Association. Part II: Symposia Papers): S348-S368.

Griesemer, J. (2000b) 'Reproduction and the reduction of genetics', in P. Beurton, R. Falk, and H-J. Rheinberger (eds.) The Concept of the Gene in Development and Evolution:

Historical and Epistemological Perspectives, 240-285. New York: Cambridge University Press.

Greisemer, J. (2000c) 'The Units of Evolutionary Transition', Selection 1-3: 67-80.

Guantes, R., Diaz-Dolunga, J. and F. Iborra (2016) 'Mitochondria and the non-genetic origins of cell-to-cell variability: More is different', Bioessays 38/1: 54-76.

Hamilton, A. and M. H. Haber (2006) 'Clades Are Reproducers', Biological Theory 1/4: 381391.

Hull, D. L. (1978) ‘A Matter of Individuality’, Philosophy of Science 45: 335-360. 
Jablonka, E. (2002) 'Information: Its Interpretation, its Inheritance, and its Sharing', Philosophy of Science 69/4: 578-605.

Kass, L. R. and J. Q. Wilson (1998) The Ethics of Human Cloning. Washington: AEI Press.

Kolers, A. (2003) 'Cloning and Genetic Parenthood', Cambridge Quarterly of Healthcare Ethics 12: $401-410$.

Kolers, A. and T. Bayne (2001) 'Are You My Mommy? On The Genetic Basis Of Parenthood', Journal of Applied Philosophy 18: 273-285.

Kolers, A. and T. Bayne (2003) 'Toward a pluralistic account of parenthood', Bioethics 17: 221242.

Laing, J. A. (2006) 'Artificial Reproduction, Blood Relatedness, and Human Identity', The Monist 89/4: 548-566.

Lewens, T. (2015) 'The Biological Foundations of Bioethics', Oxford: OUP.

Lewontin, R. C. (1998) 'The Confusion Over Cloning', in G. E. Pence (ed.) Flesh of My Flesh: The Ethics of Cloning Humans: A Reader, 129-139. Lanham, MD: Rowman \& Littlefield.

Mertes, H. (2014) 'Gamete derivation from stem cells: revisiting the concept of genetic parenthood', Journal of Medical Ethics 40: 744-7.

Mertes, H. and G. Pennings (2008) 'Embryonic Stem Cell-Derived Gametes and Genetic Parenthood: A Problematic Relationship', Cambridge Quarterly of Healthcare Ethics 17/1: 7-14.

Nuffield Council on Bioethics (2012) Novel Techniques for the Prevention of Mitochondrial Disorders: An Ethical Review. London: Nuffield Council on Bioethics.

Okasha, S. (2006) Evolution and the Levels of Selection. Oxford: OUP.

O'Malley, M. (2016) 'Reproduction Expanded: Multigenerational and Multilineal Units of Evolution', Philosophy of Science 83/5: 835-847.

Perera, F. and J. Herbstman (2011) 'Prenatal environmental exposures, epigenetics, and disease', Reproductive Toxicology 31/3: 363-373.

Rheinberger, H., Müller-Wille, S., and R. Meunier (2015) 'Gene', in E. N. Zalta (ed.) The Stanford Encyclopedia of Philosophy, Available at: $<$ http://plato.stanford.edu/archives/spr2015/entries/gene/>.

Ringler, G. (2015) 'Get Ready for Embryos From Two Men or Two Women', Time, March 18. Available at: <http://time.com/3748019/same-sex-couples-biological-children/>

Silver, L. M. and S. R. Silver (1998) 'Confused heritage and the absurdity of genetic ownership', Harvard Journal of Law and Technology 11: 593-618.

Sparrow, R. (2006) 'Cloning, Parenthood, and Genetic Relatedness', Bioethics 20: 308-318.

Sparrow, R. (2012) 'Orphaned at conception: the uncanny offspring of embryos', Bioethics 26: 173-181.

Szathmáry, E. and J. Maynard Smith (1997) 'From Replicators to Reproducers: the First Major Transitions Leading to Life', Journal of Theoretical Biology 187/4: 555-571.

Thompson, C. (2005) Making Parents: The Ontological Choreography of Reproductive Technologies. MIT Press. 